\title{
Detector DTMF Baseado em Modelagem Auto Regressiva
}

\author{
Thiago T. P. Oliveira, Leonardo S. Resende e Raimes Moraes
}

\begin{abstract}
Resumo - Este trabalho apresenta um método alternativo de detecção de tons DTMF (Dual Tone Multi-Frequential) baseado na técnica de Estimação Adaptativa dos Pólos, proposta por A. Nehorai e D. Starer [1]. O método é capaz de estimar diretamente as raízes da equação característica (i.e. os pólos) de um processo auto-regressivo que, no caso, correspondem aos valores de freqüência das senóides que compõem os tons DTMF. O detector DTMF proposto foi testado com um arquivo wave da fita de testes MITEL CM7291 e comparado com detectores baseados no algoritmo de Goertzel. Ele se mostrou mais robusto a interferências do que os detectores tradicionais, além de atender às exigências da Recomendação Q.24 da ITUT.
\end{abstract}

Palavras-Chave - detecção, sinais DTMF, processo autoregressivo.

Abstract - This work presents an alternative method of DTMF (Dual Tone Multi-Frequential) detection based on Adaptive Pole Estimation technique proposed by A. Nehorai and D. Starer [1]. It is able to estimate directly the roots of the characteristic equation (i.e. the poles) describing an autoregressive process that, in the case, correspond to the frequency values of the sine waves composing the DTMF tones. The proposed DTMF detector was tested with a wave file of MITEL CM7291 Test Tape and compared to detectors based on Goertzel algorithm. It has demonstrated to be more robust to the interferences than the traditional ones, besides to comply with ITU-T Recommendation $\mathbf{Q . 2 4}$ requirements.

Keywords - detection, DTMF signals, autoregressive process.

\section{INTRODUÇÃO}

Os tons DTMF (Dual Tone Multi-Frequential) são amplamente utilizados em telefonia e identificadores de chamada. São também utilizados por radioamadores, para controlar repetidoras à distância, e por redes de televisão, para controlar equipamentos remotos.

O sistema DTMF utiliza oito freqüências distintas transmitidas em pares, representando dezesseis dígitos diferentes. As freqüências são divididas em um grupo de freqüências baixas e um grupo de freqüências altas e o tom de cada tecla é formado por duas freqüências distintas, cada uma de um grupo. A Tabela I mostra como estas freqüências estão organizadas. As freqüências dos tons DTMF foram escolhidas de tal forma que os harmônicos e os produtos de intermodulação não causem erros no sistema. Nenhuma freqüência é múltipla de outra e a diferença ou a soma entre qualquer par de frequiências é sempre única.

T. T. P. Oliveira é Eng. de Telecomunicações da ELETROSUL Centrais Elétricas S.A. e aluno de mestrado na UFSC (GPqCom), e-mail: thiago.pains@gmail.com. Leonardo S. Resende e Raimes Moraes são professores no Departamento de Engenharia Elétrica da UFSC, laboratório GPqCom. E-mails: \{leonardo, raimes\} @eel.ufsc.br.
Tabela I. Freqüências do sistema DTMF

\begin{tabular}{|c|c|c|c|c|c|}
\hline & \multicolumn{4}{|c|}{ Freqüências Altas } \\
\hline & & $1209 \mathrm{~Hz}$ & $1336 \mathrm{~Hz}$ & $1477 \mathrm{~Hz}$ & $1633 \mathrm{~Hz}$ \\
\hline \multirow{4}{*}{ 急 } & $697 \mathrm{~Hz}$ & 1 & 2 & 3 & A \\
\hline & $770 \mathrm{~Hz}$ & 4 & 5 & 6 & B \\
\hline & $852 \mathrm{~Hz}$ & 7 & 8 & 9 & $\mathrm{C}$ \\
\hline & $941 \mathrm{~Hz}$ & $*$ & 0 & \# & $\mathrm{D}$ \\
\hline
\end{tabular}

Este trabalho aplica o algoritmo de Estimação Adaptativa dos Pólos, proposto por A. Nehorai e D. Starer, para conceber um método alternativo de detecção de sinais DTMF computacionalmente eficiente e mais robusto a interferências que os detectores tradicionalmente utilizados [1].

Este artigo está organizado da seguinte forma: a Seção I apresenta uma introdução sobre o sistema DTMF e o objetivo do trabalho; a Seção II trata das especificações recomendadas pela ITU-T; a Seção III aborda a geração e a detecção de tons; a Seção IV trata do uso da modelagem auto-regressiva para detecção de freqüências; a Seção V apresenta o algoritmo utilizado neste trabalho e suas vantagens; a Seção VI mostra os resultados da comparação entre o método de detecção proposto e o método baseado no algoritmo de Goertzel e a Seção VII traz as conclusões do trabalho.

\section{ESPECIFICAÇÕES DO SISTEMA DTMF}

As primeiras especificações para geração e detecção de tons DTMF foram elaboradas pela Bell Labs no documento TR-TSY-000181 [2]. A partir de 1988, a ITU-T estabeleceu os padrões de transmissão e detecção de sinais DTMF nas Recomendações Q.23 [3] e Q.24 [4].

Os principais requisitos de recepção são os limites toleráveis de desvio de frequiência, os níveis de potência do sinal recebido, a diferença de potência recebida entre cada uma das freqüências que compõem o dígito (twist), os tempos de duração, pausa e interrupção do sinal, a taxa de falsas detecções em sinais de fala e as condições de detecção sob interferência de eco.

A Recomendação Q.24 [4] também especifica que o tom de chamada, quando dentro das especificações, não deve interferir na detecção de um sinal DTMF e recomenda a realização de testes de imunidade a ruído. Como são muitas as possibilidades de interferência (linhas de potência, circuitos de comunicação e outros), o documento não 
especifica um valor mínimo de relação sinal-ruído sobre o qual o detector deve operar; mas estabelece que, independentemente do nível de ruído, a detecção deve ser realizada de forma a atender a todos os requisitos. Para uma referência de SNR em linhas telefônicas, a Recomendação P.830 da ITU-T [5] adota uma faixa de 15 a $45 \mathrm{~dB}$ para ruído gaussiano.

\section{GERAÇÃO E DETECÇÃO DE TONS DTMF}

\section{A. Geração de tons DTMF}

Para gerar onda senoidal digitalmente, é possível fazer uso de um filtro IIR de segunda ordem, com dois pólos complexos conjugados situados sobre o círculo unitário, descrito por

$$
H(z)=\frac{A \sin \left(2 \pi \frac{f_{0}}{f_{s}}\right)}{1-2 \cos \left(2 \pi \frac{f_{0}}{f_{s}}\right) z^{-1}+z^{-2}} .
$$

Os pólos desse filtro formam o par complexo conjugado $e^{ \pm j \omega_{0}}$, em que $\omega_{0}=2 \pi \frac{f_{0}}{f_{s}}$ é a freqüência de oscilação e $f_{s}$ a freqüência de amostragem.

A resposta desse filtro no domínio do tempo corresponde a uma onda senoidal com a freqüência $\omega_{0}$ e é descrita por

$$
y(n)=2 \cos \left(\omega_{0}\right) y(n-1)-y(n-2),
$$

sendo $y(-2)=-A \sin \left(\omega_{0}\right)$ e $y(-1)=0$.

Para gerar o tom DTMF, empregam-se dois osciladores senoidais com frequiências de oscilação programáveis; um gera a senóide do grupo de frequiências baixas e o outro gera a senóide do grupo de freqüências altas.

\section{B. Detecção de tons DTMF via DFT}

Detectar um sinal DTMF válido é uma tarefa mais complexa do que gerá-lo. Além de atender os requisitos de recepção, a detecção de DTMF deve ser ininterrupta, checando a presença de dígitos válidos continuamente e em tempo real.

De um modo geral, quando o número de componentes espectrais a serem analisadas é pequeno, a análise via DFT baseada em banco de filtros se mostra uma solução mais viável que o cálculo da FFT, pois requer um número menor de amostras a serem analisadas [6]. Este é o caso da detecção de tons DTMF, que possui apenas oito freqüências de interesse. A DFT é obtida por $[6,7,8]$

$$
X(k)=\sum_{n=0}^{N-1} x(n) W_{N}^{k n},
$$

em que $k=0,1,2, \ldots, N-1$, e $W_{N}^{k n}=e^{-j \frac{2 \pi k n}{N}}$.

$\mathrm{O}$ algoritmo de Goertzel é um método bastante eficiente e rápido, que obtém o quadrado da magnitude de $X(k)$ sem a necessidade de se realizar operações com parâmetros complexos. Esse algoritmo consiste em um banco de filtros
Tabela II. Resumo do Algoritmo de Goertzel

1. Calcular Recursivamente, para $n=0$...N:

$$
d_{k}(n)=x(n)+2 \cos \left(\frac{2 \pi f_{k}}{f_{s}}\right) d_{k}(n-1)-d_{k}(n-2)
$$

onde $d_{k}(-1)=0, d_{k}(-2)=0$ e $x(n)$ denota a amostra de entrada

2. Calcular a cada $\mathbf{N}$ amostras, $\mathrm{em} \mathbf{n}=\mathbf{N}$ :

$$
|X(k)|^{2}=d_{k}^{2}(N-1)-2 \cos \left(\frac{2 \pi k}{N}\right) d_{k}(N-1) d_{k}(N-2)+d_{k}^{2}(N-2)
$$

passa-baixas (IIR de segunda ordem), onde cada filtro extrai informações de uma determinada frequiência. As etapas do algoritmo são apresentadas resumidamente na Tabela II.

A magnitude quadrática é requerida para as oito freqüências que compõem os dígitos DTMF e também para as oito freqüências correspondentes ao segundo harmônico da cada uma das freqüências fundamentais. $O$ dígito detectado é composto pelas componentes com maior magnitude, ou seja, com maior valor de $|X(k)|^{2}$. A magnitude quadrática do segundo harmônico permitirá, durante a fase de validação, a distinção de um dígito legítimo de DTMF de um dígito falso detectado em um sinal de música ou de fala [8].

Outros métodos podem ser utilizados para detecção de DTMF. Alguns baseados na DFT e no Algoritmo de Goertzel, outros utilizando técnicas alternativas de análise em frequiência. Normalmente os métodos propostos como alternativas ao Algoritmo de Goertzel buscam otimizar algum aspecto do processo de detecção: eficiência computacional, consumo de memória, consumo de potência, robustez a interferência de fala e ruído, ou até mesmo melhorar algum aspecto da concepção do detector para alguma plataforma específica $[9,10,11]$.

\section{DETECÇÃO DE FREQÜÊNCIAS UTILIZANDO MODELAGEM AUTO-REGRESSIVA}

Modelar um sinal é, basicamente, descobrir as leis que regem sua geração e propor as equações que o descrevem. $\mathrm{O}$ uso de modelos na representação de processos estocásticos data de 1927 e consiste no fato de que uma série temporal $y(n)$, formada por observações com alto grau de correlação, pode ser gerada a partir de uma série de dados $x(n)$, estatisticamente independentes (dados não correlacionados), aplicados a um sistema linear [12].

Para um processo linear, a soma ponderada das amostras anteriores e atual da saída é igual à soma ponderada das amostras anteriores e atual de sua entrada, isto é [12],

$$
\begin{gathered}
a_{0} y(n)+a_{1} y(n-1)+\ldots+a_{p} y(n-p)= \\
b_{0} x(n)+b_{1} x(n-1)+\ldots+b_{q} x(n-q),
\end{gathered}
$$

em que $y(n)$ denota a amostra atual, $y(n-k), k=1 \ldots p$, o valor da saída a $k$-amostras atrás, $x(n)$ a amostra atual de entrada, $x(n-l), l=1 \ldots q$, o valor da entrada a $l$-amostras atrás e $a_{0} \ldots a_{p}$ e $b_{0} \ldots b_{q}$ são os parâmetros do processo. 
Se a combinação linear das amostras passadas e presente de um processo estocástico corresponde somente ao valor presente de $x(n)$, isto é, se na Equação (4), $b_{0}=1$ e $b_{1} \ldots b_{q}=0$, então essa combinação representa um processo dito ser auto-regressivo de ordem $p$ (denotado por $\operatorname{AR}(p))$ e satisfaz

$$
y(n)=-\sum_{k=1}^{p} a_{k} y(n-k)+x(n),
$$

onde $a_{1} \ldots a_{p}$ são os parâmetros do processo. Observa-se que $y(n)$ pode ser estimado da combinação linear de suas amostras de saída passadas e da amostra atual de $x(n)$.

Assumindo que a amostra atual $x(n)$ do sistema linear é desconhecida, $y(n)$ pode ser estimado somente a partir da combinação linear de $p$ amostras passadas, sendo dado por

$$
\tilde{y}(n)=-\sum_{k=1}^{p} a_{k} y(n-k) .
$$

A estimação do sinal $y(n)$ busca minimizar um sinal de erro $e(n)$, dado pela diferença entre $y(n)$ e sua estimação $\tilde{y}(n)$. Temos então

$$
e(n)=y(n)-\tilde{y}(n)=y(n)+\sum_{k=1}^{p} a_{k} y(n-k) .
$$

Para um sinal com $k$ senóides, que é o caso generalizado de um tom DTMF, a ordem mínima do modelo autoregressivo para modelagem do espectro é $2 k$. Nesse caso, cada par de pólos complexos conjugados do modelo autoregressivo equivalerá a um par de pólos complexos conjugados das senóides, e podemos estabelecer, assim, uma relação direta entre a fase de cada par de pólos conjugados e o valor da freqüência de cada senóide. $\mathrm{O}$ valor da frequiência relacionada a esse par de pólos pode ser encontrado através da expressão

$$
f=\frac{f_{s}}{2 \pi}|\arg |
$$

em que $f_{s}$ é a freqüência de amostragem e $\arg$ é a razão entre a parte imaginária e a parte real do pólo do modelo auto-regressivo representado em coordenadas retangulares, ou a própria fase do pólo em coordenadas polares.

A vantagem da utilização de modelos auto-regressivos para estimação espectral é que, ao contrário dos métodos de análise espectral baseados na Transformada de Fourier, a modelagem auto-regressiva não assume nenhuma característica para os dados da amostra que se encontram fora da janela utilizada (a DFT assume que esses dados são cíclicos). Por essa razão, o modelo auto-regresivo pode fornecer uma melhor resolução de frequiência, além disso, é possível ter estimações espectrais precisas mesmo com o uso de poucas amostras [13].

\section{MÉTOdo DE EstimaÇÃo AdAPTATIVA dos PÓlOS}

Conforme apresentamos na seção anterior, a identificação de freqüências através de um modelo auto-regressivo está associada diretamente aos valores dos pólos desse modelo.
Os métodos convencionais de predição linear, no entanto, identificam os coeficientes do polinômio característico do modelo e, só depois, determinam os valores dos pólos através de algum método de fatoração. Esse processo pode exigir uma carga computacional muito elevada se a aplicação precisar ser realizada em tempo real, pois, nesse caso, as predições devem sempre ser refeitas a conjuntos sucessivos de amostras.

Nehorai e Starer [1] propuseram um algoritmo que prediz diretamente os valores dos pólos de um modelo autoregressivo com coeficientes reais, sendo capaz de realizar eficientemente novas predições a cada novo conjunto de amostras recebido. Esse algoritmo parametriza diretamente os pólos do modelo auto-regressivo, minimizando o erro de predição através de uma busca recursiva.

Para iniciarmos a apresentação do método de estimação adaptativa dos pólos, escreveremos a Equação (7) na forma matricial, já que o propósito desta análise é uma aplicação computacional. Temos então

$$
e(n)=y(n)-\tilde{y}(n)=y(n)+\mathbf{a}^{T} \mathbf{y}(n-1),
$$

em que

$$
\mathbf{y}(n-1)=[-y(n-1),-y(n-2), \ldots,-y(n-p)]^{T}
$$

denota o vetor coluna contendo $p$ amostras passadas do sinal de saída.

Consideraremos, a partir daqui, o erro quadrático ponderado como função custo a ser otimizada, o qual pode ser descrito por

$$
\varepsilon(n)=\frac{1}{2} \sum_{i=1}^{n} \lambda^{n-i} e^{2}(i),
$$

em que $\lambda$ é o fator de ponderação. Podemos expandir a Equação (11), escrevendo o erro quadrático como

$$
\begin{aligned}
\varepsilon(n)= & \frac{1}{2} \sum_{i=1}^{n} \lambda^{n-i}\left[y^{2}(i)-2 \sum_{k=1}^{p} a_{k} y(i) y(i-k)+\right. \\
& \left.\sum_{l=1}^{p} \sum_{k=1}^{p} a_{l} a_{k} y(i-l) y(i-k)\right] .
\end{aligned}
$$

O conjunto de pólos $\theta_{q}, q=1 \ldots p$, que minimiza a função custo, deve atender à condição

$$
\frac{\partial \varepsilon(n)}{\partial \theta_{q}}=0
$$

Desenvolvendo a expressão $\frac{\partial \varepsilon(n)}{\partial \theta_{q}}$, chegamos à

$$
\frac{\partial \varepsilon(n)}{\partial \theta_{q}}=\sum_{i=1}^{n} \lambda^{n-i} e(i) \frac{\partial e(i)}{\partial \theta_{q}},
$$

em que $\frac{\partial e(i)}{\partial \theta_{q}}$ pode ser descrito, utilizando a regra da cadeia, por

$$
\frac{\partial e(i)}{\partial \theta_{q}}=\frac{\partial e(i)}{\partial a_{k}} \frac{\partial a_{k}}{\partial \theta_{q}}
$$


$\mathrm{e} \frac{\partial e(i)}{\partial a_{k}}=y(i-k)$

A manipulação algébrica do termo $\frac{\partial a_{k}}{\partial \theta_{q}}$ é necessária para que o algoritmo proposto por Nehorai e Starer seja capaz de parametrizar diretamente os pólos do modelo autoregressivo. Mostraremos posteriormente como isso é feito.

A fim de chegarmos a um conjunto de equações recursivas que estimem diretamente o valor dos pólos do modelo $\operatorname{AR}(p)$, devemos obter primeiramente a Hessiana da função custo. Para tanto, reescreveremos, utilizando novamente a regra da cadeia, a Equação (14) como

$$
\frac{\partial \varepsilon(n)}{\partial \theta_{q}}=\frac{\partial a_{k}}{\partial \theta_{q}} \frac{\partial \varepsilon(n)}{\partial a_{k}}
$$

A Hessiana da função custo pode então ser obtida através da derivada da Equação (16) em relação à $\theta_{t}$. Temos então

$$
H_{\theta_{q} \theta_{t}}(\varepsilon(n))=\left[\frac{\partial^{2} a_{k}}{\partial \theta_{q} \partial \theta_{t}} \frac{\partial \varepsilon(n)}{\partial a_{k}}+\frac{\partial a_{k}}{\partial \theta_{q}} \frac{\partial^{2} \varepsilon(n)}{\partial a_{k} \partial \theta_{t}}\right]
$$

É possível provar que o primeiro termo da Equação (17) pode ser aproximado para zero [14]. Dessa forma, podemos reescrever a Hessiana da função custo como

$$
H_{\theta_{q} \theta_{t}}(\varepsilon(n)) \cong \frac{\partial a_{k}}{\partial \theta_{q}} \frac{\partial^{2} \varepsilon(n)}{\partial a_{k} \partial \theta_{t}} .
$$

Desenvolvendo a expressão acima, chegamos a

$$
H_{\theta_{q} \theta_{t}}(\varepsilon(n))=\frac{\partial a_{k}}{\partial \theta_{q}} \sum_{l=1}^{p} y(i-l) y(i-k) \frac{\partial a_{l}}{\partial \theta_{t}},
$$

que, na forma matricial é descrito por

$$
\mathbf{H}_{\boldsymbol{\theta}}(\varepsilon(n))=\boldsymbol{\varphi}(n)\left[\sum_{i=1}^{n} \lambda^{n-i} \mathbf{y}(i-1) \mathbf{y}^{T}(i-1)\right] \boldsymbol{\varphi}^{T}(n),
$$

em que $\mathbf{H}_{\theta}(\varepsilon(n))$ é uma matriz $p \times p, \mathbf{y}(i-1)$ é um vetor coluna de dimensão $p$, definido pela Equação (10) e $\varphi(n)$ é uma matriz $p \times p$ definida por

$$
\boldsymbol{\varphi}(n)=\frac{\partial \mathbf{a}(n)}{\partial \boldsymbol{\theta}^{T}(n)} .
$$

Definindo $\psi(i)$ como o vetor coluna de dimensão $p$ descrito por

$$
\begin{aligned}
\boldsymbol{\psi}(i)=\frac{\partial e(i)}{\partial \boldsymbol{\theta}^{T}(n)} & =\frac{\partial \mathbf{a}(n)}{\partial \boldsymbol{\theta}^{T}(n)} \frac{\partial e(i)}{\partial \mathbf{a}(n)} \\
& =-\boldsymbol{\varphi}(n) \mathbf{y}(i-1),
\end{aligned}
$$

podemos escrever a Hessiana da função custo como

$$
\mathbf{H}_{\boldsymbol{\theta}}(\varepsilon(n))=\sum_{i=1}^{n} \lambda^{n-i} \boldsymbol{\psi}(i) \boldsymbol{\psi}^{T}(i) .
$$

Para estimação direta dos pólos, utilizaremos a equação generalizada para busca recursiva [14]

$$
\boldsymbol{\theta}(n+1)=\boldsymbol{\theta}(n)-[\mathbf{P}(n)] \frac{\partial \varepsilon(n)}{\partial \boldsymbol{\theta}^{T}(n)},
$$

em que $n$ denota a $n$-ésima iteração e $\mathbf{P}(n)$ uma matriz quadrada que modifica a direção da busca.

O termo $\frac{\partial \varepsilon(n)}{\partial \boldsymbol{\theta}^{T}(n)}$ pode ser escrito da seguinte maneira:

$$
\begin{aligned}
\frac{\partial \varepsilon(n)}{\partial \boldsymbol{\theta}^{T}(n)} & =\frac{\partial \mathbf{a}(n)}{\partial \boldsymbol{\theta}^{T}(n)} \frac{\partial \varepsilon(n)}{\partial \mathbf{a}(n)}=\boldsymbol{\varphi}(n)\left[\sum_{i=1}^{n} \lambda^{n-i} \mathbf{y}(i-1) e(i)\right] \\
& =\boldsymbol{\varphi}(n)\left[\lambda(n) \frac{\partial \varepsilon(n-1)}{\partial \mathbf{a}(n-1)}+\mathbf{y}(n-1) e(n)\right] .
\end{aligned}
$$

Assumindo que a iteração em $n-1$ minimiza a função custo em $n-1$, isto é, fazendo $\frac{\partial \varepsilon(n-1)}{\partial \mathbf{a}(n-1)} \cong 0$, podemos reescrever a Equação (25) como [14]

$$
\frac{\partial \varepsilon(n)}{\partial \boldsymbol{\theta}^{T}(n)}=\boldsymbol{\varphi}(n) \mathbf{y}(n-1) e(n) \text {. }
$$

Utilizando o resultado da Equação (26) e assumindo $\mathbf{P}=\left[\mathbf{H}_{\theta}(\varepsilon(n))\right]^{-1}$, a Equação (24) passa a ser escrita como

$$
\boldsymbol{\theta}(n+1)=\boldsymbol{\theta}(n)-\left[\mathbf{H}_{\boldsymbol{\theta}}(\varepsilon(n))\right]^{-1} \boldsymbol{\psi}(n) e(n) .
$$

Sendo que a Hessiana da função custo também pode ser obtida de forma recursiva, através da equação

$$
\mathbf{H}_{\boldsymbol{\theta}}(\varepsilon(n))=\lambda(n) \mathbf{H}_{\boldsymbol{\theta}}(\varepsilon(n-1))-\boldsymbol{\psi}(n) \boldsymbol{\psi}^{T}(n) .
$$

Segundo Ljung [14], para tornar mais eficientes os cálculos da Equação (27), em que a inversão matricial de $\mathbf{H}_{\theta}(\varepsilon(n))$ se faz necessária, podemos aplicar o lema de inversão de matrizes e chegarmos a

$$
\mathbf{L}(n)=\frac{\left[\mathbf{H}_{\theta}(\varepsilon(n-1))\right]^{-1} \boldsymbol{\psi}(n)}{\lambda(n)+\boldsymbol{\psi}^{T}(n)\left[\mathbf{H}_{\theta}(\varepsilon(n-1))\right]^{-1} \boldsymbol{\psi}(n)}
$$

e

$\left[\mathbf{H}_{\mathbf{0}}(\varepsilon(n))\right]^{-1}=\frac{\left[\mathbf{H}_{\mathbf{0}}(\varepsilon(n-1))\right]^{-1}-\mathbf{L}(n) \boldsymbol{\Psi}^{T}(n)\left[\mathbf{H}_{0}(\varepsilon(n-1))\right]^{-1}}{\lambda(n)}$.

Para utilizarmos as equações recursivas descritas acima, precisamos conhecer $\frac{\partial a_{k}}{\partial \theta_{q}}$. A fim de manipularmos algebricamente esse termo, escrevê-lo-emos em coordenadas polares:

$$
\theta_{q}=\rho_{q} e^{ \pm j \omega_{q}},
$$

em que $\theta_{q}$ é o $q$-ésimo par de pólos complexos conjugados do modelo, $\rho_{q}$ representa seu módulo e $\omega_{q}$ sua fase. Como visamos à aplicação na deteç̧ão de tons DTMF, que consistem em somas de senóides, não consideramos os pólos reais nesse modelo. É proposital a adoção das coordenadas polares, pois, assim, os parâmetros têm uma interpretação física diretamente relacionada às freqüências. 
A utilização das coordenadas polares permite reescrever $\frac{\partial \mathbf{a}(n)}{\partial \boldsymbol{\theta}^{T}(n)}$ como a matriz particionada

$$
\frac{\partial \mathbf{a}(n)}{\partial \boldsymbol{\theta}^{T}(n)}=\left[\frac{\partial \mathbf{a}(n)}{\partial \boldsymbol{\rho}^{T}(n)} \frac{\partial \mathbf{a}(n)}{\partial \boldsymbol{\omega}^{T}(n)}\right] .
$$

Nehorai e Starer [1] desenvolveram, a partir da fatoração do polinômio característico do modelo $\mathrm{AR}$ e da derivada desse polinômio em relação à $\rho$ e $\omega$, as seguintes expressões recursivas para os elementos das matrizes da Equação (32):

$$
\begin{gathered}
\frac{\partial a_{j}}{\partial \rho_{i}}=2 \rho_{i} \cos \left(\omega_{i}\right) \frac{\partial a_{j-1}}{\partial \rho_{i}}-\rho_{i}{ }^{2} \frac{\partial a_{j-2}}{\partial \rho_{i}}-2 \cos \left(\omega_{i}\right) a_{j-1}+2 \rho_{i} a_{j-2} \\
\frac{\partial a_{0}}{\partial \rho_{i}}=0 \text { e } \frac{\partial a_{1}}{\partial \rho_{i}}=-2 \cos \left(\omega_{i}\right) \\
\frac{\partial a_{j}}{\partial \omega_{i}}=2 \rho_{i} \cos \left(\omega_{i}\right) \frac{\partial a_{j-1}}{\partial \omega_{i}}-\rho_{i}^{2} \frac{\partial a_{j-2}}{\partial \omega_{i}}+2 \rho_{i} \sin \left(\omega_{i}\right) a_{j-1} \\
\frac{\partial a_{0}}{\partial \omega_{i}}=0 \text { e } \frac{\partial a_{1}}{\partial \omega_{i}}=2 \rho_{i} \sin \left(\omega_{i}\right)
\end{gathered}
$$

É importante observar que, nas equações acima, bem como na Equação (9), é necessário conhecer o vetor de coeficientes polinomiais a. Apesar dessa necessidade, o algoritmo proposto por Nehorai e Starer [1] apresenta a vantagem de poder obtê-lo recursivamente a partir dos pólos, através da recursão

$a_{j}(n)=a_{j}(n-1)-2 \rho_{n} a_{j-1}(n-1) \cos \left(\omega_{n}\right)+\rho_{n}^{2} a_{j-2}(n-1)$,

para $1 \leq j \leq 2 n$ e $1 \leq n \leq p / 2$, sendo $a_{0}(0)=1 \quad \mathrm{e}$ $a_{j}(n)=0$ para valores de $j$ e $n$ fora do intervalo especificado. É importante observar que essa recursão já considera a simetria dos coeficientes obtidos a partir de pares de pólos complexos conjugados.

O algoritmo de Estimação Adaptativa dos Pólos proposto por Nehorai e Starer [1] é apresentado na Tabela III. Recomenda-se iniciar o algoritmo da seguinte forma: $\left[\mathbf{H}_{\theta}(\varepsilon(n))\right]^{-1}=c \mathbf{I}$, onde $c$ é uma constante qualquer, $\mathbf{y}(0)=\psi(1)=0, \lambda(0)=0,99, \lambda(1)=0,95, \lambda(\infty)=1$.

O método convencional de obtenção de parâmetros, em que o polinômio característico é atualizado e fatorado a cada iteração, é mais exigente em termos computacionais, pois a fatoração é uma operação não linear e requer uma solução iterativa. Ao contrário do algoritmo proposto por Nehorai e Starer, o método convencional não possui um número de operações fixo [1]. Foi apresentada em [1] uma comparação numérica comprovando a eficiência computacional do algoritmo ali proposto em relação ao método convencional.

Em [1], são deduzidas também as condições de convergência do algoritmo. É demonstrado que as únicas condições que o algoritmo deve obedecer para garantir a convergência é que não hajam nem múltiplos pólos e nem pólos nulos. As condições de convergência do algoritmo(pólos distintos e não nulos) consistem em mais uma vantagem para o desenvolvimento de um detector DTMF, já que o par de senóides que compõem o tom pode
Tabela III. Algoritmo de Estimação Adaptativa dos Pólos

$e(n)=y(n)-\hat{\mathbf{a}}^{T}(n) \mathbf{y}(n-1)$
$\mathbf{L}(n)=\frac{\left[\mathbf{H}_{\boldsymbol{\theta}}(\varepsilon(n-1))\right]^{-1} \boldsymbol{\psi}(n)}{\lambda(n)+\boldsymbol{\psi}^{T}(n)\left[\mathbf{H}_{\boldsymbol{\theta}}(\varepsilon(n-1))\right]^{-1} \boldsymbol{\psi}(n)}$
$\left[\mathbf{H}_{\boldsymbol{\theta}}(\varepsilon(n))\right]^{-1}=\frac{\left[\mathbf{H}_{\boldsymbol{\theta}}(\varepsilon(n-1))\right]^{-1}-\mathbf{L}(n) \boldsymbol{\Psi}^{T}(n)\left[\mathbf{H}_{\boldsymbol{\theta}}(\varepsilon(n-1))\right]^{-1}}{\lambda(n)}$
$\boldsymbol{\theta}(n+1)=\boldsymbol{\theta}(n)-\mathbf{L}(n) e(n)$
- Obtenção de $\hat{\mathbf{a}}(n+1)$
- Obtenção de $\frac{\partial \hat{\mathbf{a}}(n+1)}{\partial \boldsymbol{\theta}(n+1)}$
$\mathbf{y}(n)=[-y(n),-y(n-1), \ldots,-y(n-p+1)]^{T}$
$\boldsymbol{\psi}(n+1)=\left[\frac{\partial \hat{\mathbf{a}}(n+1)}{\partial \boldsymbol{\theta}(n+1)}\right] \mathbf{y}(n)$
$\lambda(n+1)=\lambda(\infty)-(\lambda(\infty)-\lambda(n)) \lambda(0)$

ser descrito por dois pares distintos de pólos complexos conjugados. Além disso, as características computacionais do algoritmo demonstram que ele é apto a realizar estimações em tempo real. Baseados nisso, desenvolvemos um detector de tons DTMF utilizando o algoritmo de Estimação Adaptativa dos Pólos e o submetemos a testes de detecção, imunidade a ruído e o comparamos com os métodos tradicionais de detecção.

\section{RESUltados}

O detector desenvolvido foi simulado no ambiente MATLAB e, para verificar se ele atende à Recomendação Q.24 da ITU-T, foi submetido ao teste MITEL CM7291 [15]. Para avaliação do seu desemepenho diante de sinais contaminados com ruído, ele foi comparado a um detector baseado no algoritmo de Goertzel [16]. Os seguintes parâmetros são configuráveis no detector desenvolvido:

- Ordem do sistema (número de pólos): interfere diretamente na carga computacional exigida, no número de falsas detecções e na imunidade à interferências.

- Tamanho do buffer: interfere diretamente nos tempos de recepção e de guarda do sinal e na imunidade a interferências.

- Índice de rejeição do sinal: interfere inversamente no número de falsas detecções e diretamente na imunidade à interferências.

Foram testados detectores com diferentes ordens, variando de 4 a 10, cada um com uma configuração apropriada de tamanho de buffer e índice de rejeição do sinal, estabelecida de forma empírica. Foi verificado que, para o teste da MITEL CM7291, que é contaminado com ruído com SNR na ordem de $24 \mathrm{~dB}$, é necessário utilizar pelo menos 6 pólos complexos, ou seja, para que sejam atendidos os requisitos exigidos pela ITU-T, é necessário configurar o detector, no mínimo, para ordem 6. Comprovamos que, para um sinal similar; porém sem ruído, qualquer configuração do detector satisfaz a norma [17]. 
Para compararmos o desempenho do detector baseado no algoritmo de Estimação Adaptativa dos Pólos com um detector baseado no algoritmo de Goertzel, quando ambos são submetidos à sinais contaminados com ruído, utilizaremos como referência o detector de DTMF desenvolvido por Trajkovic [16], que é baseado no algoritmo de Goertzel e foi testado sendo submetido um conjunto de 10000 pulsos do dígito "1" para uma série de valores de SNR, em que a Probabilidade de Detecção foi definida pela razão entre os tons detectados corretamente e o total de tons submetidos à detecção. Se o detector apresenta uma probabilidade de mais de $90 \%$ de detecção, é considerado aprovado no teste. Os resultados dos testes do detector desenvolvido, comparado ao detector baseado no algoritmo de Goertzel são apresentados na Fig. 1.

Baseado nos parâmetros de Trajkovic para validação do detector, o detector baseado no algoritmo de Estimação Adaptativa dos Pólos apresentou um limiar de operação de $26 \mathrm{~dB}$ para um modelo de predição linear de ordem 4, de 15 $\mathrm{dB}$ para um modelo de ordem 6 , de $9 \mathrm{~dB}$ para um modelo de ordem 8 e de $8 \mathrm{~dB}$ para um modelo de ordem 10. Como o limiar de operação para um detector DTMF é $15 \mathrm{~dB}$ [16], qualquer um dos detectores testados, com exceção do modelo de ordem 4, estariam habilitados. Quando modelos de ordem 8 ou 10 são utilizados, o detector desenvolvido consegue limiares de operação de $9 \mathrm{~dB}$ e de $8 \mathrm{~dB}$, respectivamente.

Podemos constatar então que o detector proposto é mais robusto em ambientes ruidosos que o detector baseado no algoritmo de Goertzel. É possível constatar também, através da Fig. 1 que, o desempenho do detector proposto utilizando modelos AR de ordens 8 e 10 é bastante superior ao detector baseado no algoritmo de Goertzel, apresentando uma quantidade de detecções considerável (cerca de 90\%) para uma SNR de $8 \mathrm{~dB}$, valor para o qual o detector baseado no algoritmo de Goertzel já não detecta sequer $50 \%$ dos tons.

\section{CONCLUSÃO}

A detecção de tons DTMF tem sido tradicionalmente realizada por meio do algoritmo de Goertzel, que consiste num método baseado em DFT. Outras alternativas têm sido propostas no sentido de melhorar alguns aspectos da detecção. $\mathrm{O}$ uso de métodos baseados em predição linear pode fornecer detectores mais robustos, especialmente em meios ruidosos, já que a análise em freqüência através da predição linear apresenta melhor resolução em frequiência e pode obter bons resultados com poucas amostras. O custo computacional para se obter um espectro através de predição linear, no entanto, é consideravelmente maior que o custo computacional requerido pelo algoritmo de Goertzel.

O uso do algoritmo de Estimação Adaptativa dos Pólos mostrou ser uma alternativa capaz de reduzir a carga computacional da detecção via modelagem auto-regressiva e de fornecer uma boa modelagem espectral. Através desse método, obtivemos um detector com parâmetros facilmente configuráveis, com boa robustez e melhor desempenho em sinais contaminados com ruído do que as aplicações baseadas no algoritmo de Goertzel utilizadas para comparação.

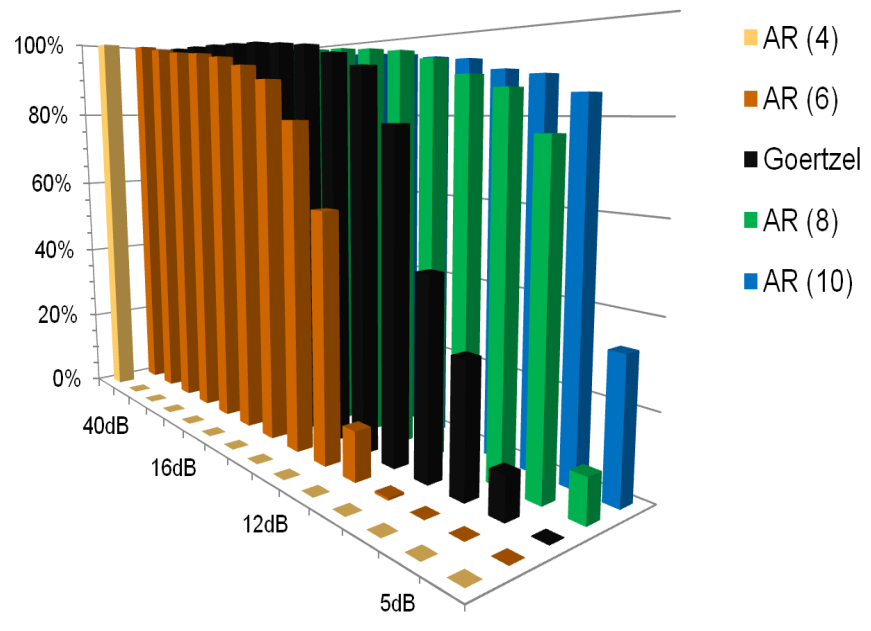

Figura 1. Teste de SNR

\section{REFERÊNCIAS}

[1] A. Nehorai, and D. Starer, "Adaptive pole estimation," IEEE Trans. Acoust., Speech, Signal Processing, Vol. ASSP-38, pp. 825-838, 1990.

[2] Bell Communications Research Technical Reference TR-TSY000181, "Dual-Tone Multifrequency Receiver Generic Requirements for End-to-End Signaling Over Tandem-Switched Voice Links", Issue 1, 1987.

[3] ITU-T Blue Book, "Recommendation Q.23: Technical Features of Push-Button Telephone Sets", Geneva,1988.

[4] ITU-T Blue Book, "Recommendation Q.24: Multi-frequency PushButton Signal Reception”, Geneva,1988.

[5] ITU-T, "Recommendation P.830: Subjective Performance Assessment of Telephone-Band and Wideband Digital Codecs", Geneva, 1996.

[6] S. M. Kuo and B. H. Lee, "Real Time Digital Signal Processing", John Wiley \& Sons Ltd., 2001, cap. 9

[7] S. M. Kuo and W. Gan, "Digital Signal Processors. Architectures, Implementations, and Applications", Pearson Prentice Hall, 2005, cap. 7

[8] Texas Instrument, "DTMF Tone Generation and Detection: An Implementation Using the TMS320C54x", SPRA096A, 2000.

[9] R. Becker and J. Mulder, "SIGFRED: A Low-Power DTMF and Signaling Frequency Detector", IEEE Journal of Solid-State Circuits, v. 26, n. 7, p. 1027-1037, Jul. 1991.

[10] A.V.N.S. Anjani and B. Yegnanarayana "An Autoregressive Model Based Approach for DTMF Detection", Proc. National Conference on Communication, Delhi, Jan. 2000.

[11] V. Emiya, L.F.C. Pessoa, D. Vallot, D. Melles. "Generic Tone Detection using Teager-Kaiser Energy Operators on the StarCore SC140 core". Freescale application note AN2384/D, Aug. 2003.

[12] S. Haykin; "Adaptive Filter Theory", Prentice Hall, Englewood Cliffs, NJ, second edition, 1991, cap 1-5.

[13] J. Markhoul; "Linear prediction: A tutorial review", Proc. IEEE 63, April 1975, pp. 561-580..

[14] L. Ljung; "System Identification - Theory For the User", 2nd edition, PTR Prentice Hall, Upper Saddle River, N.J., 1999.

[15] MITEL TECHNICAL DATA; "Tone Receiver Text Cassette CM7291", 1980.

[16] M. Trajkovi; "Performance analysus of the DTMF detector based on the Goertzel's algorithm", 14th Telecommunications Forum TELFOR, Serbia, Belgrado, November 2006.

[17] T.T.P. Oliveira, "Utilização da Técnica de Estimação Adaptativa de Pólos para Detecção de Tons DTMF", Dissertação de Mestrado, UFSC, 2009. 